\title{
Case-control study of childhood leukaemia and cancer in Scotland: findings for neonatal intramuscular vitamin $\mathrm{K}$
}

\author{
Patricia A McKinney, Edmund Juszczak, Elizabeth Findlay, Katrina Smith
}

\begin{abstract}
Objective: To test the hypothesis of an association between neonatal intramuscular vitamin $\mathrm{K}$ and childhood leukaemia and other cancers.

Design: Population based case-control study with data abstracted from hospital records.

Setting: Scotland.

Subjects: Children aged 0-14 years resident in Scotland from 1991-4 and diagnosed with leukaemia (150), lymphomas (46), central nervous system tumours (79), a range of other solid tumours (142), and a subset of acute lymphoblastic leukaemia (129). Controls were 777 children matched for age and sex, providing 417 matched sets (360 triplets and 57 pairs) for analysis.
\end{abstract}

Main outcome measure: Odds ratios for the risk of childhood leukaemia and cancer and intramuscular vitamin $\mathrm{K}$ versus a combined group of oral doses, none, and no record. Results are given for information recorded in medical notes and data supplemented by hospital policy.

Results: Odds ratios based on medical record abstractions showed no significant positive association for leukaemias (odds ratio 1.30; 95\% confidence interval 0.83 to 2.03 ), acute lymphoblastic leukaemia (1.21; 0.74 to 1.97$)$, lymphomas $(1.06 ; 0.46$ to 2.42$)$, central nervous system tumours $(0.74 ; 0.40$ to 1.34$)$, and other solid tumours $(0.59 ; 0.37$ to 0.96$)$. There was no association with acute lymphoblastic leukaemia in children aged 1 to 6 years. Imputation of exposure from hospital policy gave similar results. Adjustment for deprivation and type of delivery moved risk estimates closer to unity for all major diagnostic groups.

Conclusions: The observation of an increased risk of childhood leukaemia and cancer associated with intramuscular vitamin $\mathrm{K}$ is not confirmed by this independent population based study.

\section{Introduction}

The issue of whether the prophylactic administration of intramuscular vitamin $\mathrm{K}$ to neonates increases the risk of childhood cancer continues to be of importance both clinically and in the public health arena. Prophylactic use of vitamin $\mathrm{K}$ to reduce the incidence of vitamin $\mathrm{K}$ deficiency bleeding has been weighed against the possible risk of developing a malignancy during childhood. $^{1}$ After the original observations from Bristol associating intramuscular doses with a raised risk of leukaemia ${ }^{2}$ there has been a dearth of epidemiological evidence to support the original findings. Studies from Sweden, ${ }^{3}$ Denmark, ${ }^{4}$ the United States, ${ }^{5}$ Germany, ${ }^{6}$ and England ${ }^{7}$ have all failed to demonstrate any excess of childhood leukaemia or cancer in children who received intramuscular vitamin $\mathrm{K}$. The findings of these investigations, however, remain inconclusive because of lack of power ${ }^{5}$ or presumed rather than documented exposure to vitamin $\mathrm{K}^{34}$ The alternative use of medical notes is not ideal and the considerable difficulties of assessment of exposure are acknowledged.$^{67}$ Recording may be incomplete, and to compensate for this by imputing exposure according to the hospital policy at the time of birth will introduce misclassification as policy does not always equate with delivery of the drug. ${ }^{7}$ To clarify the issue of vitamin $\mathrm{K}$ and fulfil the requirement for results from large well designed studies we conducted an analysis of data specifically collected on vitamin $\mathrm{K}$.

Childhood cancer comprises a heterogeneous group of malignancies which are generally unique to childhood and clinically and pathologically distinct from adult cancers. The rarity of the disease can present difficulties for epidemiological research when the presence of small numbers necessitates the aggregation of biologically distinct diagnostic subgroups, therefore large studies are required to investigate putative risk factors. The Scottish case-control study of childhood leukaemia and other cancers was undertaken to identify risk factors for childhood cancer in the Scottish population. ${ }^{8}$ One component was the collection of data from hospital maternity records to study obstetric and perinatal risk factors. Our paper presents the results of testing the specific hypothesis of an association between intramuscular vitamin $\mathrm{K}$ and childhood cancer for the diagnostic subgroups of leukaemias, acute lymphoblastic leukaemia, lymphomas, central nervous system tumours, and other solid tumours.

\section{Patients and methods}

This population based case-control study defined cases as children (0-14 years) diagnosed with leukaemia and cancer during 1991-4 while they were living in Scotland, the childhood population of which at the 1991 census was 959 268. All diagnoses were
See editorial by von Kries and pp 178, 184, 189, 230

Information and Statistics Division, Common Services Agency for the National Health Service in Scotland, Trinity Park House, Edinburgh EH5 3SQ

Patricia A McKinney, principal researcher Edmund Juszczak, senior statistician Elizabeth Findlay, research nurse Katrina Smith, senior administrator

Correspondence to: Dr McKinney p.a.mckinney@ leeds.ac.uk

BMJ 1998;316:173-7 
pathologically confirmed and completeness of case ascertainment was optimised by cross checking with the Scottish Cancer Registration Scheme and the National Register of Childhood Tumours in Britain. ${ }^{9}$ Controls matched on age (to within 1 month), sex, and health board of residence were randomly selected from all eligible children registered for primary care within each health board. An optimum of two controls per case were included and methodological details are published elsewhere. ${ }^{8}$

Mothers were approached and interviewed with consent from the consultant clinician or general practitioner. At interview mothers gave their signed permission for the researchers to abstract their medical notes. Medical records were seen from 76 hospitals and maternity units throughout Scotland relating to births from 1976 to 1994 . For the small number of births in England and Wales (3.4\%) records were obtained by post.

\begin{abstract}
ion of notes
Two trained researchers recorded information from obstetric, delivery, and neonatal records, including nursing notes, on to a highly structured standard form, specifically designed to collect data on vitamin $\mathrm{K}$ and validated in a previous study of childhood leukaemia and obstetric and perinatal risk factors. ${ }^{7}$ The senior midwife abstracter (EF) ensured consistency and data quality through a programme of continual monitoring, coding checks, and duplicate abstractions. Demographic details of the mother were collected at interview and additional data were obtained for a wide range of variables including method of delivery and admission to a special care baby unit.
\end{abstract}

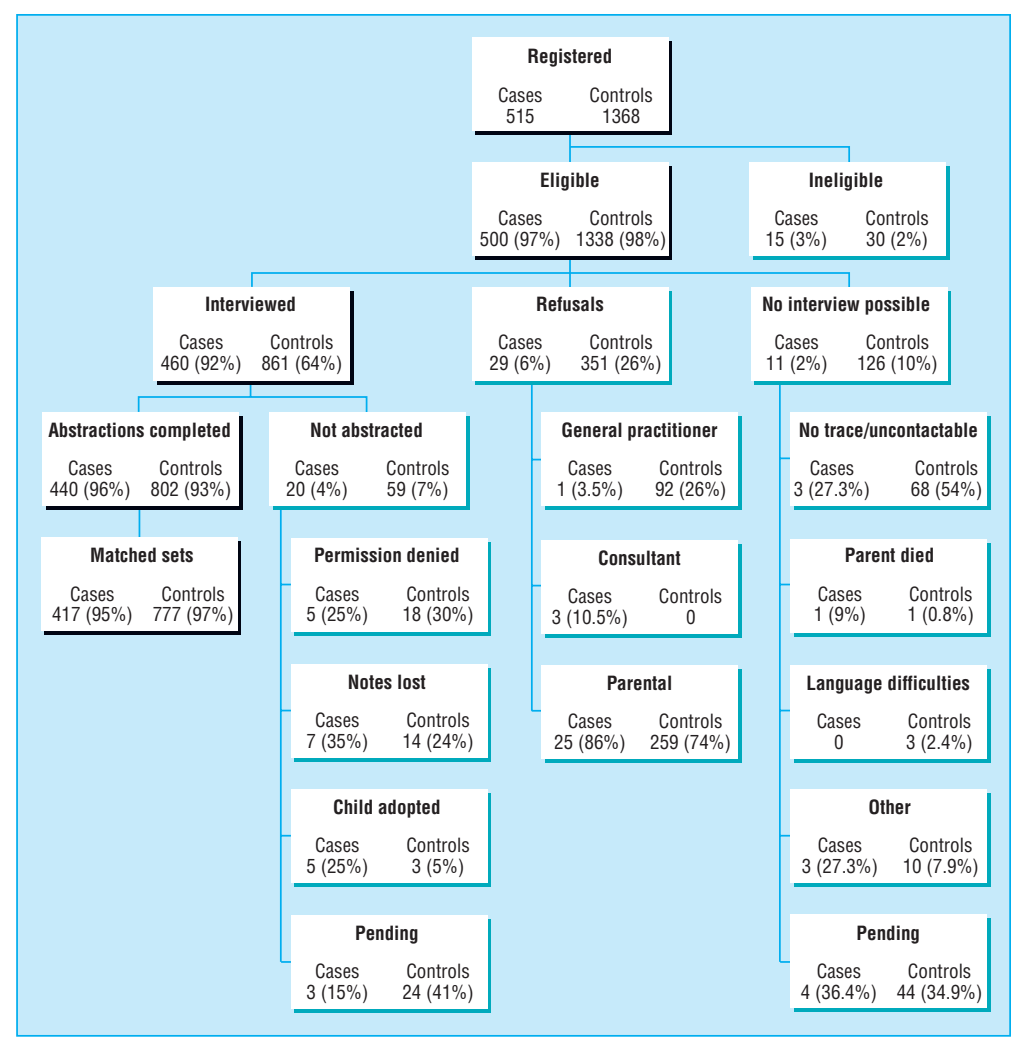

Numbers of cases and controls registered, numbers of interviews undertaken, and proportions of mothers with completed abstractions of obstetric notes
Vitamin $\mathrm{K}$ was recorded as given or definitely not given only when this was mentioned in the notes and similarly the route of administration-intramuscular, oral, or not recorded. Information collected on the vitamin $\mathrm{K}$ policies for hospitals delivering over 500 babies annually was provided year by year (1976-91) by Dr E Hey and the Scottish Neonatal Network. These data came from two sources and were validated by abstraction of a sample of medical records. The current study further validated the data through consultations with hospital pharmacies and senior labour room midwives. Prophylaxis was defined as either universal intramuscular, universal oral, selective intramuscular, or uncertain policy and was used to impute exposure for the analysis groups defined as: recorded-entirely on the basis of what was recorded in the medical notes, but in instances when the route was not recorded this was derived from hospital policy; and imputed-as for "recorded" but with the addition of imputing intramuscular and oral doses when the hospital of delivery had a policy of universal intramuscular or oral prophylaxis and nothing was written in the medical notes.

Most babies will have received $1 \mathrm{mg}$ of phytomenadione (Konakion) intramuscularly, although a small number of hospitals gave $0.5 \mathrm{mg}$ doses. All analyses compared intramuscular vitamin $\mathrm{K}$ with oral doses, vitamin K recorded as given with "not known" route of administration, and not given and no record combined, ensuring comparability with other studies $^{267}$ and reflecting the absence of any risk for oral doses.

\section{Analysis}

Diagnostic subsets of data as defined by the Birch and Marsden classification ${ }^{10}$ comprised leukaemias, lymphomas, central nervous system tumours, and other solid tumours (neuroblastomas, retinoblastomas, renal, hepatic, bone and germ cell tumours, soft tissue sarcomas, and carcinomas). Two subgroups of the leukaemias-acute lymphoblastic leukaemia and acute lymphoblastic leukaemia diagnosed at age 1-6 years (12-83 months)-were also investigated to ensure comparability with published literature. ${ }^{67}$

The dataset for statistical analysis was restricted to matched sets, and information was lost for 23 cases that lacked controls and 25 controls without a matched case. These were proportionally distributed across the diagnostic groups. A standard case-control analysis ${ }^{11}$ of 417 matched case-control sets (360 triplets and 57 pairs) was performed with STATA, ${ }^{12}$ which permits variable matching ratios. Odds ratios, 95\% confidence intervals, and $\mathrm{P}$ values were calculated by using conditional logistic regression.

To account for potential confounding factors for the exposure variable (intramuscular vitamin $\mathrm{K}$ ) odds ratios were adjusted for type of delivery (normal vaginal, assisted (forceps and ventouse), and caesarean). Childhood leukaemia and central nervous system tumours have been associated with increased affluence $^{13} 14$ so adjustment was also made for social class at the time of birth. Each child was allocated a deprivation score by taking the address at birth and, via the validated postcode, assigning an area measure of deprivation on the basis of census data according to Carstairs and Morris. ${ }^{15}$ The three categories ranged 
from the most affluent to the most deprived, the remainder being referred to as standard.

\section{Results}

The figure details the number of children registered and shows that a high proportion of those interviewed (96\% cases, 93\% controls) had their obstetric records reviewed. The refusal rate for cases and controls was $6 \%$ and $26 \%$, respectively, with a further group identified for which no interview was possible; this latter group were not considered true refusals. A total of 1194 case and control abstractions contributed to the matched sets for analysis, and table 1 gives a breakdown by diagnostic group indicating the principal subgroups.

With medical records as the primary source of information the case-control distribution of vitamin $\mathrm{K}$ and its route of administration can be seen in table 2 for the two main analytical groups. In the "recorded group" $8.2 \%$ of cases and $9.3 \%$ of controls were included as receiving intramuscular vitamin $\mathrm{K}$ but the route was imputed. In the "imputed group" an additional 53 cases and 79 controls were added to the intramuscular imputed group from the "nil written in notes" category. For 107 (25.7\%) cases and 188 (24.2\%) controls no hospital policy was available for any imputation, including births of 17 cases and 23 controls in English hospitals.

Crude and adjusted odds ratios for recorded and imputed data are shown in table 3 by diagnostic group. None of the raised odds ratios reach significance. Negative associations were present for the "other solid tumours" with a significant crude odds ratio. Adjustment for deprivation and type of delivery gave odds ratios closer to 1 except for the lymphomas. The numbers of discordant or informative sets are generally reduced for the analysis of imputed data, resulting in wider confidence intervals.

A subset of acute lymphoblastic leukaemia in children aged 1-6 years (90 cases, 174 controls) was also analysed. The odds ratio (95\% confidence interval) for recorded data was 1.08 ( 0.60 to 1.94 ) on the basis of 50 discordant sets and for imputed data was 1.25 (0.68 to 2.28) on the basis of 48 discordant sets. The adjusted
Table 1 Numbers of note abstractions for cases, controls, and matched sets by diagnostic group

\begin{tabular}{lrrrr} 
Diagnostic group & No of cases & No of controls & Matched triplets & Matched pairs \\
\hline Leukaemias: & 150 & 284 & 134 & 16 \\
\hline Acute lymphoblastic leukaemia & 129 & 247 & 118 & 11 \\
\hline Lymphomas & 46 & 86 & 40 & 6 \\
\hline Central nervous system tumours & 79 & 141 & 62 & 17 \\
\hline Other solid tumours: & 142 & 266 & 124 & 18 \\
\hline Neuroblastomas & 39 & 71 & 32 & 7 \\
\hline Retinoblastomas & 7 & 14 & 7 & - \\
\hline Renal tumours & 25 & 47 & 22 & 3 \\
\hline Hepatic tumours & 4 & 8 & 4 & - \\
\hline Malignant bone tumours & 14 & 27 & 13 & 1 \\
\hline Soft tissue sarcomas & 25 & 47 & 22 & 3 \\
\hline Germ cell tumours & 17 & 32 & 15 & 2 \\
\hline Carcinomas & 10 & 18 & 8 & 2 \\
\hline Other neoplasms & 1 & 2 & 1 & - \\
\hline Overall total & 417 & 777 & 360 & 57 \\
\hline
\end{tabular}

Table 2 Frequency of data from 417 cases and 777 controls by category of exposure to neonatal vitamin $\mathrm{K}$

\begin{tabular}{|c|c|c|c|c|}
\hline \multirow[b]{2}{*}{ Category of exposure } & \multicolumn{2}{|c|}{ Recorded* (\%) } & \multicolumn{2}{|c|}{ Imputed† (\%) } \\
\hline & Cases & Controls & Cases & Controls \\
\hline \multicolumn{5}{|l|}{ Vitamin K given: } \\
\hline Intramuscular (from notes $\ddagger$ ) & $157(37.6)$ & $303(39.0)$ & $157(37.6)$ & $303(39.0)$ \\
\hline Intramuscular (route imputed $\neq$ ) & $34(8.2)$ & $72(9.3)$ & $34(8.2)$ & $72(9.3)$ \\
\hline Intramuscular (imputed $\neq$ ) & NA & NA & $53(12.7)$ & $79(10.1)$ \\
\hline Oral (from notes) & $12(2.9)$ & $31(4.0)$ & $12(2.9)$ & $31(4.0)$ \\
\hline Oral (route imputed) & 0 & 0 & 0 & 0 \\
\hline Oral (imputed) & NA & NA & 0 & $2(0.3)$ \\
\hline Route not known & $42(10.1)$ & $77(9.9)$ & $42(10.1)$ & $77(9.9)$ \\
\hline Vitamin $\mathrm{K}$ not given & $19(4.6)$ & $22(2.8)$ & $19(4.6)$ & $22(2.8)$ \\
\hline Nil written in notes & $153(36.7)$ & $272(35.0)$ & $100(24.0)$ & $191(24.6)$ \\
\hline
\end{tabular}

$\mathrm{NA}=$ not applicable.

${ }^{*}$ On basis of written reports in hospital notes with route of delivery imputed from hospital policy. tOn basis of written reports plus imputation of dose from hospital policy. f"Exposed" group.

results gave odds ratios of 1.16 (0.62 to 2.15) for recorded data and 1.33 (0.70 to 2.53) for imputed data.

\section{Discussion}

The population based Scottish case-control study of childhood leukaemia and cancer has gathered a comprehensive set of information to investigate risk

Table 3 Odds ratios for intramuscular vitamin $\mathrm{K}$ given in neonatal period by diagnostic group

\begin{tabular}{|c|c|c|c|c|c|}
\hline Diagnostic group & $\begin{array}{c}\text { No }(\%) \text { of } \\
\text { cases }\end{array}$ & No $(\%)$ of controls & Odds ratio $(95 \% \mathrm{Cl})$ & Discordant sets & $\begin{array}{c}\text { Adjusted odds ratio* } \\
(95 \% \mathrm{CI})\end{array}$ \\
\hline \multicolumn{6}{|c|}{ Leukaemias $(n=150)$ : } \\
\hline Recorded & $82(54.7)$ & $139(48.9)$ & $1.30(0.83$ to 2.03$)$ & 88 & $1.23(0.77$ to 1.97$)$ \\
\hline \multicolumn{6}{|c|}{ Acute lymphoblastic leukaemia $(n=129)$ : } \\
\hline Recorded & $71(55.0)$ & $126(51.0)$ & $1.21(0.74$ to 1.97$)$ & 72 & $1.17(0.70$ to 1.97$)$ \\
\hline Recorded & $17(37.0)$ & $31(36.0)$ & 1.06 (0.46 to 2.42$)$ & 25 & 1.70 (0.59 to 4.95$)$ \\
\hline Imputed & $23(50.0)$ & $43(50.0)$ & $1.03(0.46$ to 2.32$)$ & 26 & 1.55 (0.49 to 4.85$)$ \\
\hline \multicolumn{6}{|c|}{ Central nervous system tumours $(n=79)$ : } \\
\hline Recorded & $33(41.8)$ & $68(48.2)$ & $0.74(0.40$ to 1.34$)$ & 48 & 1.07 (0.55 to 2.09$)$ \\
\hline Imputed & $41(51.9)$ & $84(59.6)$ & $0.64(0.33$ to 1.24$)$ & 40 & 1.04 (0.49 to 2.22) \\
\hline
\end{tabular}

${ }^{*}$ Adjusted for deprivation category (affluent, standard, deprived) and type of delivery (normal, assisted, caesarean). $\dagger P=0.033$. 
factors for childhood malignancies, with specific attention being paid to experiences during the prenatal and neonatal period. Data are available from interviews, but our study derived independent information for a range of variables, including neonatal vitamin $\mathrm{K}$ prophylaxis, from medical notes. The traditional problems of maternal recall bias associated with interview based studies were therefore avoided.

\section{Specific strengths of study}

In comparison with previously published studies the Scottish study has particular strengths. The cases are highly representative of the underlying population, with $83 \%$ included compared with $66 \%$ in the study by Von Kries. ${ }^{6}$ The number of cases analysed in the Scottish study is greater than that in previous similar investigations. ${ }^{5-7}$ No previous study has documented risks for separate groups of childhood solid tumours, specifically brain tumours. Golding's original findings suggested a risk for all childhood cancers and particularly for leukaemia. ${ }^{2}$ Separate diagnostic groups of childhood cancer are likely to have distinguishable aetiologies, and therefore our study examined the most biologically plausible subsets. Our results for childhood central nervous system tumours are new. The absence of risk in any subgroup increases the likelihood that intramuscular vitamin $\mathrm{K}$ has no influence on the development of childhood cancer.

One of the problems inherent in case-control study design is the potential for recruitment bias. The overall refusal rate for interviews was $6 \%$ for cases and $26 \%$ for controls, which reflected the strenuous efforts to interview first choice controls and compares favourably with a previous similar study. ${ }^{6}$ The geographical pattern and age distribution of refusals for cases and controls was similar, showing no evidence of bias in this respect.

A very high proportion of records was abstracted for both cases and controls, despite the fact that the births of interest spanned a 19 year period going back to 1976 . Because of the length of the study period and its national coverage a large number of maternity hospitals and units gave access to records, and for births in England and Wales notes were sent to the study centre. The data are therefore representative of hospital and maternity unit deliveries across Scotland. This is ideal to examine the issue of vitamin $\mathrm{K}$ because cases and controls were not selected according to hospital of birth as in Golding's original study, in which the hospital policy was not necessarily universally representative. $^{2}$

The use of a highly structured and validated recording form, identical with that used in an independent study by Roman and colleagues ${ }^{16}$ was the key to the systematic recording of data and reduced the possibility of introducing bias into the abstraction procedure. This was undertaken by two trained members of staff and the data subjected to consistency checks during the course of the study to ensure data quality. The abstracters were not always blind to case-control status, but it is unlikely this resulted in biased recording, specifically for vitamin $\mathrm{K}$, because of the large numbers of variables requiring attention for abstraction. The overall proportions of cases and controls in which nothing was recorded in the notes were similar for both recorded and imputed exposure, which suggests no systematic bias was present associated with either overrecording or underrecording.

\section{Overcoming problems of defining exposure}

A major difficulty experienced by studies of medical records is the lack of information actually written up in the notes in relation to vitamin $\mathrm{K}^{2.5-7} \mathrm{It}$ is accepted that many babies for whom no record was made will have received doses, but there is no reason to suspect this is more likely to have happened to cases than controls. The availability of hospital policy details can be used to supplement recorded data, but the absolute levels of exposure to vitamin $\mathrm{K}$ are likely to be an underestimate. To examine this issue fully and allow for the two sources of information, separate analyses were performed. Firstly, the examination of what appeared in the hospital notes resulted in about a third of the sample contributing nil from the notes. These "recorded" data were augmented by information derived from hospital policy for the route of administration, a procedure justified and implemented in other studies. ${ }^{267}$

To overcome the problem of entirely missing data, exposure has been imputed from hospital policy. A second analysis was therefore conducted in which the absence of a written record (given or definitely not given) intramuscular doses were imputed when hospital policy was for universal prophylaxis. This took recorded data as the primary source and increased the proportion of those babies deemed as exposed to intramuscular vitamin $\mathrm{K}$. The unexposed comparison group comprised all those who received oral doses as well as those with no administration and those with no record. This aggregation allowed for the uncertainties in the non-exposed and, by virtue of its heterogeneity, is likely to include babies who received intramuscular vitamin K. Most importantly, it is inconceivable that this misclassification was subject to case-control bias. In addition, the inclusion of oral vitamin $\mathrm{K}$ will have had a minimal effect due to the small proportion $(<5 \%)$ administered via this route. As a test of a specific hypothesis, the exposed category was considered to be as precisely estimated as possible from this type of study.

Studies of rare diseases such as childhood cancer can lack power to detect increased risk. Overall this study had $89 \%$ power to detect an odds ratio of 1.5 at the $5 \%$ level of significance, assuming two controls per case, and a $60 \%$ prevalence of intramuscular vitamin $\mathrm{K}$ exposure. This compares very favourably with other studies $^{6}$ and generates confidence in the study findings.

Certain variables were considered as potential confounders. Type of delivery was selected a priori as a known correlate of vitamin $\mathrm{K}$ administration, and a proxy for social class was taken from the residential address at the time of birth. Admission to a special care baby unit had the same effect as type of delivery. Adjustment for confounding failed to alter either the significance or the magnitude of the crude odds ratios in any notable way. Apart from the lymphomas all the adjusted odds ratios tended towards unity. For the lymphomas these were slightly increased as a result of the influence of the broad distribution of the social class categories, none of which were independently associated with lymphomas. 
- Debate continues over the role neonatal intramuscular administration of vitamin $\mathrm{K}$ may have in the subsequent development of childhood cancer

- Most previous epidemiological studies have failed to demonstrate an overall significant association

- No significant risk is evident from a population based study in Scottish children that used data from hospital records

- No relation was found for leukaemias, central nervous system tumours, or any other subgroup of childhood malignancy

Acute lymphoblastic leukaemia occurs most commonly in young children and for those in the 1-6 year age group findings have been conflicting. ${ }^{6}{ }^{16}$ Our results are consistent with the observations of Roman et al, who failed to find a significantly raised risk in this subgroup. ${ }^{16}$ Von Kries and colleagues explained their increased risk as due to chance.

The debate over the role intramuscular vitamin $\mathrm{K}$ may have in causing childhood cancer may be moving towards a conclusion. ${ }^{17}$ Our large, well designed, and carefully conducted study contributes a set of results consistent with previous studies failing to demonstrate an association between neonatal intramuscular vitamin $\mathrm{K}$ and childhood leukaemia and cancer.

We are grateful to all the people who have helped with our study and who are too numerous to be named separately. The support and collaboration of the clinicians from the UK Children's Cancer Study Group (UKCCSG) has been invaluable. Interviewers and all study staff at the Information and Statistics Division of the NHS in Scotland, especially Bea Jardine and Nadine Russell, are thanked in addition to the Cancer Surveillance Group and the directors of the Regional Cancer Registries and their staff. Consultants, general practitioners, and medical staff, in particular medical records staff, in hospitals throughout Scotland have provided extensive assistance. Scotland is a participating centre in the United Kingdom Childhood Cancer Study (UKCCS). Dr $\mathrm{E}$ Hey provided data on the policies of vitamin $\mathrm{K}$ administration for Scottish maternity hospitals. All participating families are thanked for making this research possible.

Contributors: PAM, who coordinates the Scottish case control study of childhood leukaemia and cancer, initiated and managed the specific study of vitamin K. EJ conducted the statistical analysis. EF abstracted data from obstetric notes and monitored data quality. KS supervised the data processing and validation. All contributors discussed the results and their interpretation. The paper was written by PAM with contributions from EJ, EF, and KS. PAM is guarantor for the study.

Funding: The Scottish Office, Chief Scientist Office (SODH), Scottish Power plc, Scottish Nuclear Limited, and Scottish Hydro-Electric plc. The UKCCS is sponsored and administered by the United Kingdom Coordinating Committee on Cancer Research (UKCCCR).

Conflict of interest: None.

1 Draper G, McNinch A. Vitamin K for neonates: the controversy. BMJ $1994 ; 308: 867-8$

2 Golding J, Greenwood R, Birmingham K, Mott M. Childhood cancer, intramuscular vitamin $\mathrm{K}$, and pethidine given during labour. $B M \mathrm{~J}$ 1992;305:341-6.

3 Eklund H, Finnstrom, O, Gunnarskog J, Kallen B, Larsson Y. Administration of vitamin $\mathrm{K}$ to new-born infants and childhood cancer. $B M J$ 1993:307:89-91.

4 Olsen JH, Hertz H, Blinkenberg K, Verder H. Vitamin K regimens and the incidence of childhood cancer in Denmark. BMJ 1994;308:895-6.

5 Klebanoff MA, Read JS, Mills JL, Shiono PH. The risk of childhood cancer after neonatal exposure to vitamin K. N Engl J Med 1993;329:905-8.

6 Von Kries R, Gobel U, Hachmeister A, Kaletsch U, Michaelis J. Vitamin K and childhood cancer: a population based case-control study in Lower Saxony, Germany. BMJ 1996; 313:199-203.

7 Ansell P, Bull D, Roman E. Childhood leukaemia and intramuscular vitamin K: findings from a case-control study. BMJ 1996;313:204-5.

8 McKinney PA, Smith K, Findlay E. The Scottish case-control study of childhood leukaemia and cancer: methodology and environmental measures of exposure. Health Bull (Edinb) 1995;53:222-9.

9 Stiller CA, Allen MB, Ebtock EM. Childhood cancer in Britain: the National Registry of Childhood Tumours and incidence rates 1978-87. Eur J Cancer 1995;202:8-34.

10 Birch JM, Marsden HB. A classification scheme for childhood cancer. Int J Cancer 1987;40:620-4.

11 Breslow NE, Day NE. Statistical methods in cancer research. Vol 1. The analysis of case-control studies. Lyons: IARC Scientific Publications, 1980. (Publication No 32.)

12 Stata Stata Statistical Software: release 5.0. College Station, TX: Stata, 1997.

13 McKinney PA, Ironside JW, Harkness EF, Arango JC, Doyle D, Black RJ. Registration and epidemiology of childhood brain tumours in Scotland 1975-1990. Br J Cancer 1994;70:973-9.

14 Doll R. The epidemiology of childhood leukaemia. $J R$ Statist Soc A 1989;3:341-51,

15 Carstairs V, Morris R. Deprivation and health in Scotland. Aberdeen: Aberdeen University Press, 1991

16 Roman E, Ansell P, Bull D. Leukaemia and non-Hodgkin's lymphoma in children and young adults: are prenatal and neonatal factors important determinants of disease. Br J Cancer 1997;76:406-15.

17 Zipursky A. Vitamin K at birth (editorial). BMJ 1996;313:179-80.

(Accepted 3 October 1997)

\section{A memorable patient \\ Another paper lost}

She was a pretty, fair haired girl of 16 . She and her parents had just returned from a disastrous skiing holiday in her father's native Austria. Before leaving she had noticed thirst and polyuria for a fortnight. While on holiday she had abdominal pain and a normal appendix was removed. After the operation the blood glucose concentration was $18 \mathrm{mmol} /$ litre. She was started on twice daily beef soluble insulin. On her return to Britain she had been troubled by episodes of hypoglycaemia. Examination was normal.

The hypoglycaemia became even more troublesome and the insulin dose was reduced at each clinic attendance. When the dose was very low her father asked permission to take her to a faith healer, which he did. She subsequently came off insulin. Follow up blood glucose estimations were normal and a glucose tolerance test a year after that was normal. Nothing further was heard of her.
Two years ago an elderly man under the care of another doctor beckoned me over during a ward round. He reminded me about his daughter who was by then 37 . She was well and had had three successful pregnancies with no trace of diabetes. Her helpful general practitioner looked up her notes but there were no old letters about her. A random glucose test showed a concentration of $5 \mathrm{mmol} /$ litre. The records department was asked to find the old notes. In such a case the notes were either microfilmed and often left in random order so that they could not readily be traced, or left to rot in a damp corner of a basement. Despite numerous attempts to find them they have never reappeared.

Temporary diabetes is a rare bird, and it would have been nice to be able to document this case adequately.

Nicholas Mayne, retired physician, Cheltenham 